\title{
Proteins induced by telomere dysfunction and DNA damage represent biomarkers of human aging and disease
}

\author{
Hong Jianga,b, Eric Schifferc, Zhangfa Songa, Jianwei Wanga, Petra Zürbigc, Kathrin Thedieck, Suzette Moes ${ }^{d}$, \\ Heike Bantele, Nadja Saale, Justyna Jantosc, Meiken Brecht' ${ }^{f}$, Paul Jenö ${ }^{d}$, Michael N. Halld, Klaus Hagerf, \\ Michael P. Mannse, Hartmut Heckerg, Arnold Ganserh, Konstanze Döhneri, Andrzej Bartkej, \\ Christoph Meissnerk, Harald Mischakc, Zhenyu Jua,l, and K. Lenhard Rudolpha,m
}

\begin{abstract}
anstitute of Molecular Medicine and Max Planck Research Group on Stem Cell Aging and 'Department of Internal Medicine III, University of Ulm, 89081

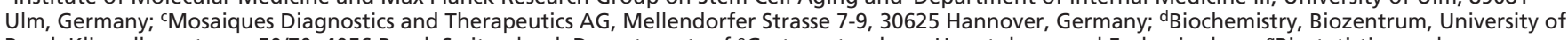
Basel, Klingelbergstrasse 50/70, 4056 Basel, Switzerland; Departments of ${ }^{\circ}$ Gastroenterology, Hepatology, and Endocrinology, ${ }^{9 B i o s t a t i s t i c s, ~ a n d ~}$ ${ }^{h}$ Hematology, Hemostasis, Oncology, and Stem Cell Transplantation, Hannover Medical School, 30625 Hannover, Germany; fDepartment of Geriatric Medicine, Henriettenstiftung, D-30171 Hannover, Germany; jPhysiology and Internal Medicine, Southern Illinois University School of Medicine, Springfield, IL 62794; ' Department of Forensic Medicine, University Hospital Schleswig Holstein, Kahlhorststrasse 31-35, 23562 Lübeck, Germany; and 'Institute of Laboratory Animal Sciences and Max-Planck-Partner Group on Stem Cell Aging, Chinese Academy of Medical Sciences, Beijing 100864, China
\end{abstract}

Edited by Titia de Lange, The Rockefeller University, New York, NY, and approved June 11, 2008 (received for review February 17, 2008)

Telomere dysfunction limits the proliferative capacity of human cells by activation of DNA damage responses, inducing senescence or apoptosis. In humans, telomere shortening occurs in the vast majority of tissues during aging, and telomere shortening is accelerated in chronic diseases that increase the rate of cell turnover. Yet, the functional role of telomere dysfunction and DNA damage in human aging and diseases remains under debate. Here, we identified marker proteins (i.e., CRAMP, stathmin, EF-1 $\alpha$, and chitinase) that are secreted from telomere-dysfunctional bone-marrow cells of late generation telomerase knockout mice (G4mTerc ${ }^{-l-}$ ). The expression levels of these proteins increase in blood and in various tissues of aging $\mathrm{G}_{\mathrm{mTerc}} \mathrm{mT}^{-/}$mice but not in aging mice with long telomere reserves. Orthologs of these proteins are up-regulated in late-passage presenescent human fibroblasts and in early passage human cells in response to $\gamma$-irradiation. The study shows that the expression level of these marker proteins increases in the blood plasma of aging humans and shows a further increase in geriatric patients with aging-associated diseases. Moreover, there was a significant increase in the expression of the biomarkers in the blood plasma of patients with chronic diseases that are associated with increased rates of cell turnover and telomere shortening, such as cirrhosis and myelodysplastic syndromes (MDS). Analysis of blinded test samples validated the effectiveness of the biomarkers to discriminate between young and old, and between disease groups (MDS, cirrhosis) and healthy controls. These results support the concept that telomere dysfunction and DNA damage are interconnected pathways that are activated during human aging and disease.

senescence $\mid$ EF- $1 \alpha \mid$ personalized therapy | stem cells | CRAMP

$\mathbf{T}^{\mathrm{c}}$ elomeres cap the chromosome ends and prevent the activation of DNA-damage checkpoints that induce cell-cycle arrest (senescence) or apoptosis $(1,2)$. Telomere shortening occurs during human aging and chronic diseases (3). Mutations in the enzyme telomerase lead to telomere shortening, impaired tissue maintenance, and a shortened lifespan in both humans (4-7) and mice (8-11). These observations indicate that telomere shortening can impair organ maintenance and shorten lifespan. However, the actual contribution of dysfunctional telomeres to natural human aging and diseases remains under debate. Accumulation of senescent cells has been detected in the skin of aging humans (12) and primates (13) but not in other organs such as muscle (14) or liver (15). In aging telomerase knockout mice $\left(\mathrm{mTerc}^{-/-}\right)$, telomere dysfunction is associated with a decline in stem cell function, impaired organ maintenance, and a shortened lifespan (8-11). Yet,
$\mathrm{mTerc}^{-1-}$ mice do not show an accumulation of senescent cells (11). There is emerging evidence that senescent cells can be cleared in vivo by induction of apoptosis (11) and immune responses (16). Moreover, the detection of senescence is cell-cycle dependent (17). Therefore, the impact of telomere dysfunction on aging might be underestimated by experiments trying to detect senescent cells. In addition, the detection of senescent cells in vivo is technically challenging, impeding its implementation as a clinical marker.

In vitro studies on human cells indicate that low levels of telomere dysfunction precede cellular senescence in culture (18). The identification of marker proteins that indicate low levels of telomere dysfunction in presenescent cells could help to determine the influence of telomere dysfunction and DNA damage on human aging and disease. In addition, the identification of such markers is of clinical interest because there is a growing population of humans that suffers from disease phenotypes associated with aging.

\section{Results}

Identification of Biomarkers of Aging Telomere Dysfunctional Mice. Late generations of telomerase knockout mice $\left(\mathrm{G} 4 \mathrm{mTerc}{ }^{-/-}\right)$do not show an accumulation of senescent cells but yet develop aging phenotypes associated with telomere dysfunction (8-11). We reasoned that this mouse model represents a good experimental system in which to identify proteins induced by telomere dysfunction in presenescent cells. To identify easily detectable markers, we analyzed secreted peptides in supernatant of short-term cultures (4 h) of total bone marrow cells from 2- vs. 12-month-old mTerc ${ }^{+/+}$and G4mTerc ${ }^{-1-}$ mice by using a proteomics approach, capillary electrophoresis-time of flight-mass spectrometry (CE-TOF-MS). Statistical analysis followed by machine-learning procedures identified and validated a set of peptides that distinguished telomere dysfunc-

Author contributions: H.J. and K.L.R. designed research; H.J., E.S., Z.S., J.W., P.Z., K.T., S.M. N.S., J.J., M.B.,P.J., M.N.H. K.H., M.P.M., A.G., A.B., C.M., H.M., and Z.J. performed research, H.J., E.S., H.B., and K.D. contributed new reagents/analytic tools; H.J., E.S., Z.S., J.W., P.Z., K.T., S.M., and H.H. analyzed data; and H.J., E.S., Z.S., and K.L.R. wrote the paper.

Conflict of interest statement: K.L.R. has recently submittted a patent application for the discovered biomarkers that are described in this work.

This article is a PNAS Direct Submission.

bPresent address: Kidney Disease Centre, The First Affiliated Hospital, College of Medicine, Zhejiang University, Hangzhou 310003, China.

mTo whom correspondence should be addressed at: Department of Molecular Medicine and Max Planck Research Group on Stem Cell Aging, University of Ulm, Albert-EinsteinAllee 11, 89081 Ulm, Germany. E-mail: lenhard.rudolph@uni-ulm.de.

This article contains supporting information online at www.pnas.org/cgi/content/full/ 0801457105/DCSupplemental.

() 2008 by The National Academy of Sciences of the USA 
Fig. 1. Identification of biomarkers of aging telomeredysfunctional mice. $(A)$ The pictures show contour plots of the three-dimensional CE-MS of polypeptides secreted into the medium from bone-marrow cells of 2- or 12month-old $\mathrm{mTerc}^{+/+}$and $\mathrm{G}^{\prime} \mathrm{mTerc}^{-1-}$ mice. Molecular mass $(0.8-20 \mathrm{kDa})$ is plotted against CE migration time (20-70 min). MS signal intensity is plotted on the $z$ axis. Note that the expression of the peptides increases in culture medium of bone-marrow cells derived from 12-
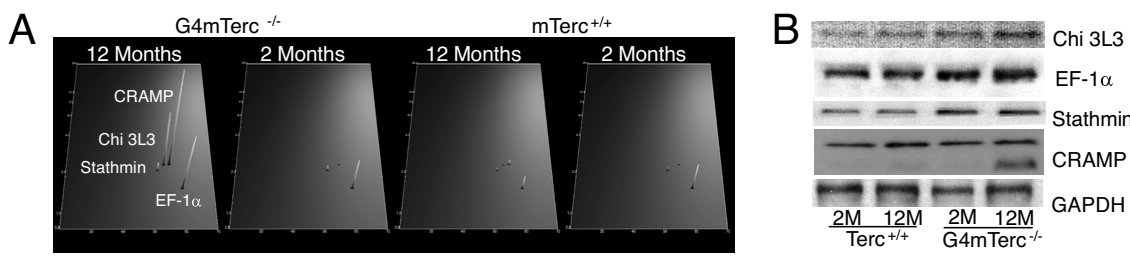

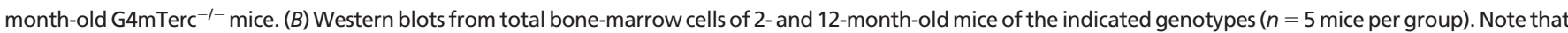
CRAMP protein shows two bands; the lower band represents the active, cleaved form of the protein, which was up-regulated in $12-$ month-old G4mTerc ${ }^{-1-}$ mice.

tional mice $\left(\mathrm{G} 4 \mathrm{mTerc}{ }^{-/-}\right)$from wild-type mice $\left(\mathrm{mTerc}^{+/+}\right)$and 12-month-old from 2-month-old mice with high accuracy [supporting information (SI) Fig. S1 $A-E]$.

Next, we identified a set of peptides by tandem massspectrometry sequencing analysis. Among them, four peptides showed increased expression in 12-month-old G4mTerc ${ }^{-1-}$ mice compared with the other three mouse cohorts $(n=5$ mice per group, Fig. 1A). The up-regulation of these markers in aged $\mathrm{G} 4 \mathrm{mTerc}{ }^{-1-}$ mice was confirmed in a blinded test set of 26 mice; 9 of $15 \mathrm{G} 4 \mathrm{mTerc}^{-1-}$ mice showed an up-regulation of the marker peptides compared with 1 of 11 mice of the other three cohorts (specificity, 91\%; sensitivity, 60\%) (data not shown). Western-blot analysis on mouse bone-marrow cells confirmed the up-regulation of these marker proteins in aging G4mTerc ${ }^{-1-}$ mice (Fig. $1 B$ and Fig. S2 $A$ and $B$ ).

The sequenced peptides were fragments derived from the following proteins: (i) Cathelicidin-related antimicrobial protein (CRAMP), which is activated during innate immune responses and protects against bacterial infection but has not been associated with aging (19), (ii) Chitinase 3-like protein 3 (Chi3L3), which belongs to the chitinase gene family and is activated in innate immune responses $(20,21)$ (a member of this family has been associated with chondrocyte aging and arthritis (22)), (iii) Elongation factor $1 \alpha$ $(\mathrm{EF}-1 \alpha)$, which controls translational protein synthesis and is up-regulated during senescence of human fibroblasts $(23,24)$, and (iv) stathmin which controls microtubule stability, cell motility, and mitosis (25).

Whereas mRNA expression of the identified markers was similar in 2-month-old G4mTerc ${ }^{-1-}$ and mTerc $^{+/+}$mice (Fig. $2 A-F$ ), an up-regulation of the markers was detected in various organs of 12-month-old G4mTerc ${ }^{-1-}$ mice but not in 12- or 24-month-old mTerc $^{+/+}$mice (Fig. $2 A-F$ ). Some of the proteins (CRAMP, Chi3L3) were up-regulated in all analyzed organs (kidney, liver, lung, brain, spleen, heart), whereas others (EF-1 $\alpha$, stathmin) showed organ specificity. Immunohistochemistry (IHC) confirmed the increased expression of the marker proteins in tissues of aging G4mTerc ${ }^{-1-}$ mice but not in mTerc ${ }^{+/+}$mice (Table S1, Fig. S2C, and Fig. S3). In addition, an up-regulation of all markers was observed in the blood plasma of 12-month-old G4mTerc ${ }^{-/-}$mice but not in 12-month-old mTerc ${ }^{+/+}$mice (Fig. $2 G$ ). CRAMP levels and chitinase enzyme activity showed a moderate up-regulation in 24-month-old $\mathrm{mTerc}^{+/+}$mice but not as significant as in 12month-old G4mTerc ${ }^{-/-}$mice. There was no reduction in the expression of the protein markers in long-lived mouse mutants (growth hormone receptor knockout mice, Ames dwarf mice) compared with wild-type mice at the age of 2-19 months (Fig. S4 $A-D)$. Together, these results indicated that we had identified a set of marker proteins that is associated with the aging of telomeredysfunctional mice.
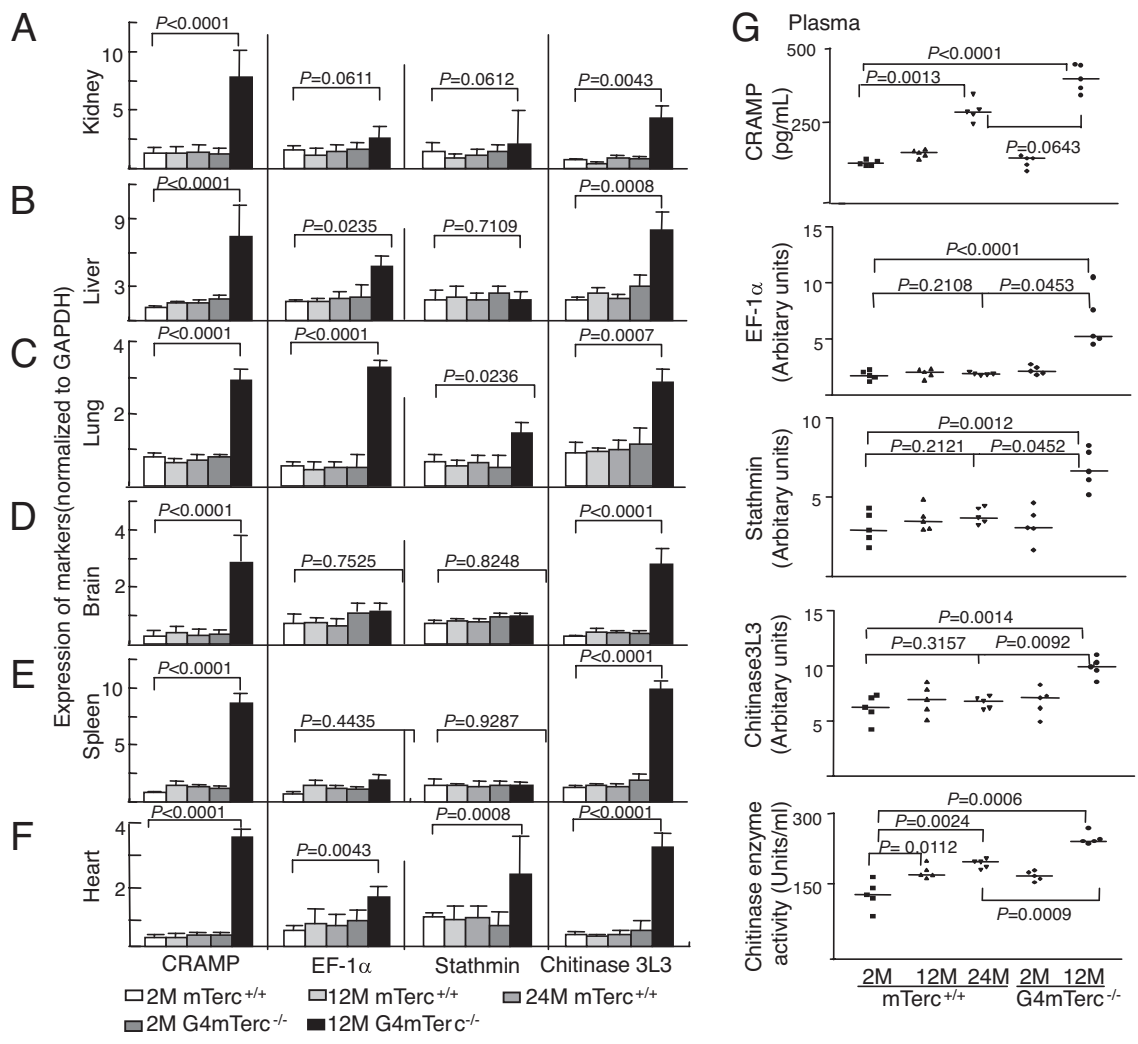

Fig. 2. Up-regulation of marker proteins in organs and plasma of aging telomere-dysfunctional mice. $(A-F)$ The histograms show the mRNA expression of the indicated biomarker relative to GAPDH in the indicated organs. The analysis was conducted on $2-, 12-$, and 24-month-old $\mathrm{mTerc}^{+1+}$ mice and on 2- and 12-monthold G4mTerc ${ }^{-1-}$ mice ( $n=5$ mice per group). The bars show mean values. Error bars show standard deviation. (G) The dot plots show the expression of the marker proteins and chitinase enzyme activity in plasma of 2-, 12-, and 24-month-old mTerc ${ }^{+/+}$mice, and of 2- and 12-month-old G4mTerc ${ }^{-1-}$ mice ( $n=5$ mice per group). The lines show mean values. 
A
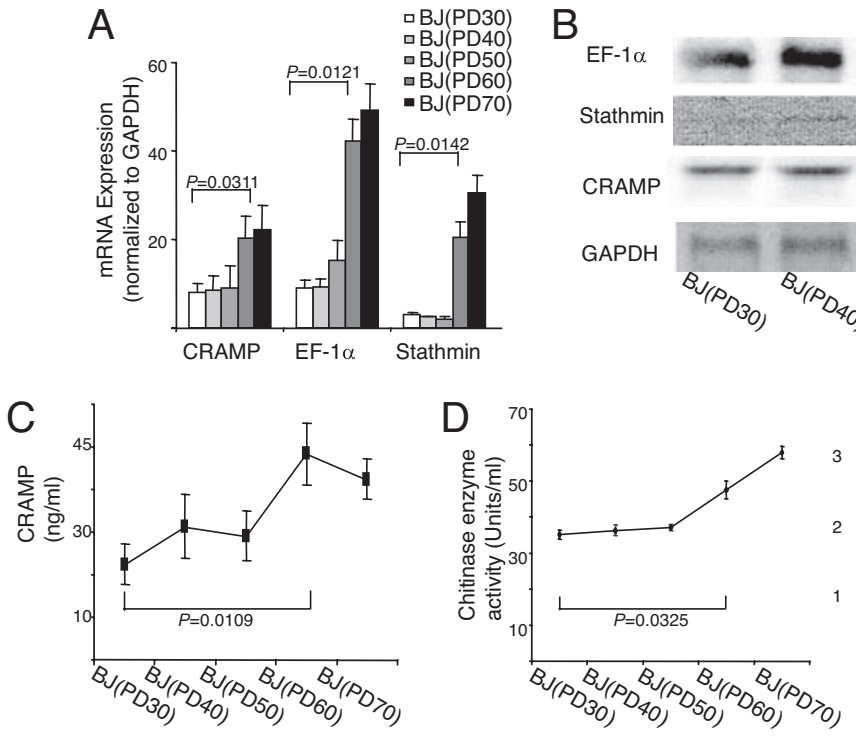

$\mathrm{F}$
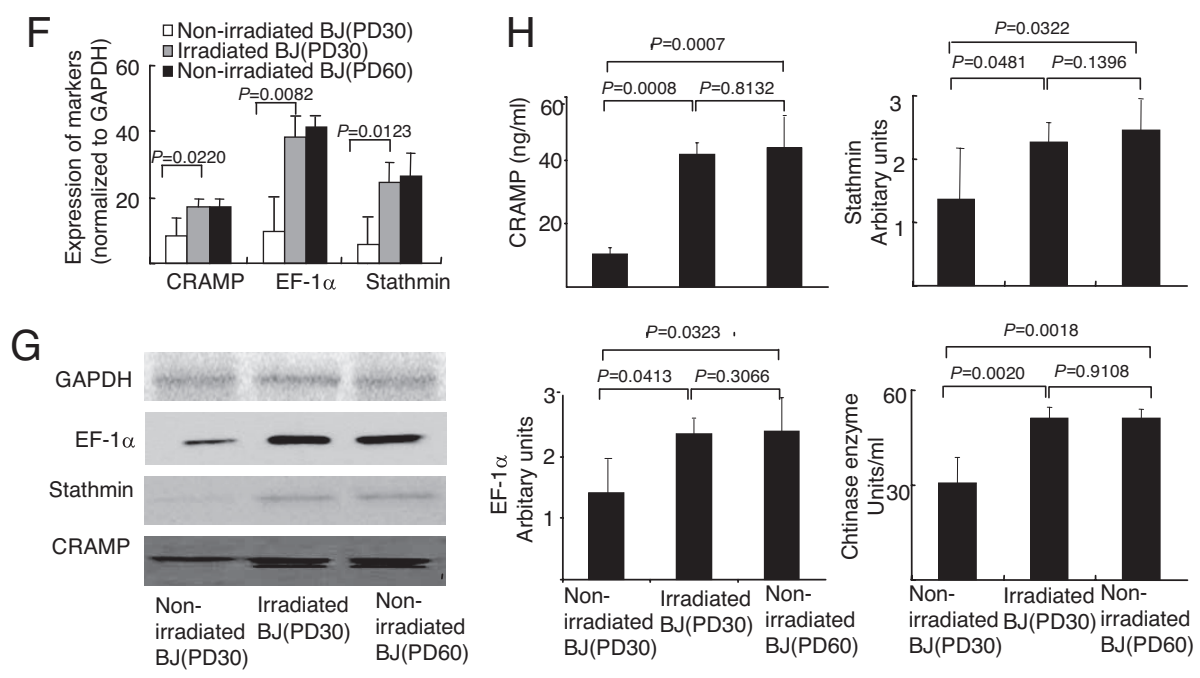

Fig. 3. Secreted proteins of telomeredysfunctional mice are overexpressed in presenescent human cells and in response DNA damage. BJ fibroblasts were used for the analyses. Under our laboratory conditions, the proliferation of BJ cells slowed down at PD60 (presenescence). The cells were fully senescent at PD70. (A) The histogram shows mRNA expression in BJ cells at the indicated PD ( $n=3$ repeat experiments). The bars show mean values; error bars show standard deviation. (B) Representative Western blots on the expression of marker proteins in BJ cells at the indicated PD $(n=3$ repeat experiments). (C-E) The histograms show protein expression ( $C$ and $E$ ) and enzyme activity $(D)$ of the indicated markers in culture medium incubated for $4 \mathrm{~h}$ on $\mathrm{BJ}$-cells at the indicated PD ( $n=3$ repeat experiments). The dots show mean values; the error bars show standard deviation. Note that the expression of marker proteins increases in presenescent BJ-cells at PD60. ( $F$ ) The histogram shows mRNA expression of the indicated biomarkers relative to GAPDH in irradiated (4 $\mathrm{h}$ after 4-Gy $\gamma$-irradiation) and nonirradiated BJ-cells at the indicated PD ( $n=3$ repeat experiments). The bars show mean values; error bars show standard deviation. (G) Representative Western blots on the expression of marker proteins in irradiated (4 $\mathrm{h}$ after 4-Gy $\gamma$-irradiation) and nonirradiated $\mathrm{BJ}$-cells at the indicated $\mathrm{PD}(n=3$ repeat experiments). (H) The histograms show protein expression (CRAMP, stathmin, $\mathrm{EF}-1 \alpha$ ) and enzyme activity (chitinase) in culture medium incubated for $4 \mathrm{~h}$ on irradiated (4-Gy $\gamma$-irradiation) and nonirradiated BJcells at the indicated PD.

\section{Secreted Proteins of Telomere-Dysfunctional Mice are Overexpressed} in Presenescent Human Cells and in Response to DNA Damage. Antibodies were available for three human orthologs (CRAMP, EF-1 $\alpha$, stathmin). The human ortholog for mouse Chi3L3 has not yet been identified, but it is possible to measure chitinase enzyme activity across different species. Under our laboratory conditions, human BJ fibroblasts become fully senescent at population doubling (PD) 70 as indicated by a near complete lack of BrdU incorporation after a 2-h labeling (data not shown). A significant increase of the mRNA and protein expression of CRAMP, EF- $1 \alpha$, and stathmin was first detectable in presenescent BJ fibroblasts at PD60 (Fig. $3 A$ and $B$ and Fig. S5 $A$ and $B$ ). In addition, presenescent cells at PD60 showed increased secretion of CRAMP, stathmin, EF- $1 \alpha$, and chitinases (Fig. $3 C-E$ ). Previous studies have shown that presenescent human cells show low levels of telomeredysfunction (18). In agreement with the assumption that telomeric DNA-damage induced the expression of the identified marker proteins, a significant up-regulation of the marker proteins was also seen in early passage BJ cells $4 \mathrm{~h}$ after $\gamma$-irradiation (4 Gy), both intracellularly (Fig. $3 F$ and $G$ and Fig. S5 $C$ and $D$ ) and in the culture medium (Fig. $3 H$ ). Together, these data indicated that the identified marker proteins were induced in human cells in response to telomere dysfunction and DNA damage.
Proteins Induced by Telomere Dysfunction and DNA Damage Show Increased Expression During Human Aging. In human blood plasma, the expression levels of CRAMP $(P<0.0001)$, EF-1 $\alpha(P=$ $0.0004)$, and stathmin $(P<0.0001)$ and the level of chitinase enzyme activity $(P=0.0004)$ were increased in unaffected, old individuals living in an elderly home $(n=20$; mean age, $85 \pm 8.1$ years) compared with unaffected, young individuals $(n=31$; mean age, $30 \pm 3.8$ years) (Fig. $4 A-D$ ). There was a further increase in the plasma levels of CRAMP $(P=0.0007)$ and EF- $1 \alpha$ $(P=0.0115)$ and in chitinase enzyme activity $(P=0.0216)$ in hospitalized geriatric patients $(n=72$; mean age, $73 \pm 8.5$ years $)$ (Fig. $4 A-D$ ), indicating that increasing plasma levels of the marker proteins correlated with aging and aging-associated diseases. Multivariate analysis revealed that CRAMP-protein level and chitinase enzyme activity had the best discriminatory power $($ combination score $=0.34 \times$ CRAMP $+0.07 \times$ chitinase) (Table S2) and the combination of these two markers had the highest statistical power to discriminate the three groups (Fig. 4E). In our collectives, these markers showed a better correlation with aging and aging-associated disease compared with the interleukin-6 (IL-6) plasma level (Fig. 4F), which is one of the classical biomarkers of human aging (26-28). ROC-curve analysis confirmed that the marker combination had a higher specificity and sensitivity to discriminate unaffected young and 


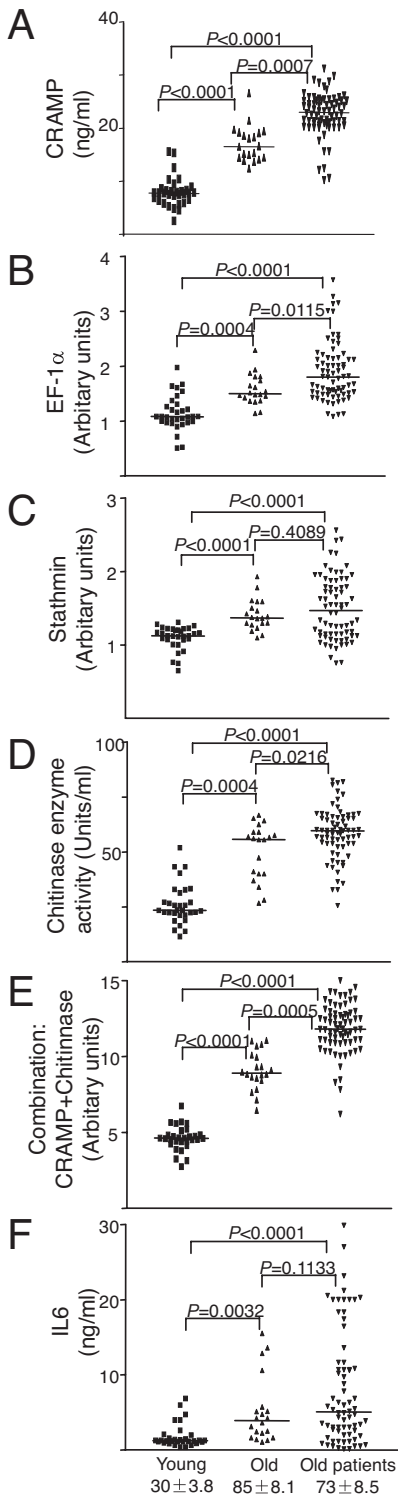

G

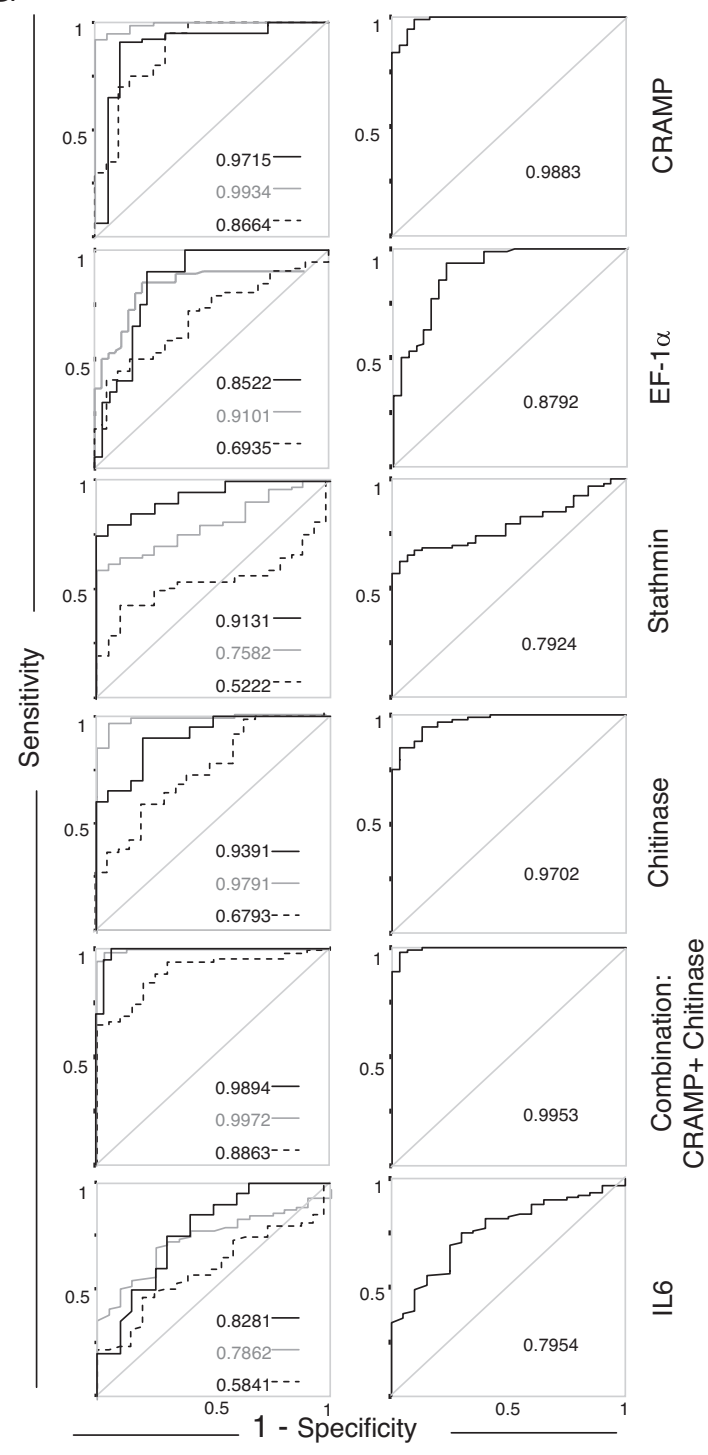

$\mathrm{H}$

$\frac{8}{4}$
- Young vs.Old persons
Young persons vs.Old patients Young persons vs.Old

Fig. 4. Proteins induced by telomere dysfunction and DNA damage show increased expression during human aging. $(A-F)$ The dot plots show the concentration of the indicated marker proteins in human blood plasma as measured by ELISA for CRAMP $(A), \operatorname{EF}-1 \alpha(B)$, and stathmin (C). (D) The dot plot shows chitinase enzyme activity in blood plasma. Note that all four marker proteins were up-regulated in unaffected aged individuals living in an elderly home (old; $n=20$; mean age, $85 \pm 8.1$ years) and more pronounced in hospitalized geriatric patients (old patients; $n=72$; mean age, $73 \pm 8.5$ years) compared with young individuals (young; $n=31$; mean age, $30 \pm 3.8$ years). (E) The dot plot shows a combined analysis of the best two markers (CRAMP, chitinase) according to the regression analysis (Table S2) on the same set of samples. $(F)$ The dot plot shows IL-6 level in plasma of the indicated cohorts. (G) The histograms show ROC analyses of the marker proteins comparing the indicated cohorts. $(H)$ The histograms show ROC analyses of the marker proteins comparing young individuals to the combined cohort of old individuals consisting of unaffected old individuals and geriatric patients $(n=92)$. Note that the levels of CRAMP, EF- $1 \alpha$, and chitinase enzyme activity correlate better with human aging than IL-6. old individuals and geriatric patients (Fig. $4 G$ and $H$ ). In line with the plasma data, IHC revealed increased expression of CRAMP, EF- $1 \alpha$, and stathmin in postmortem liver biopsies of aged humans ( $n=5$; mean age, $76 \pm 7.6$ years $)$ compared with younger individuals ( $n=6$; mean age, $21 \pm 7.7$ years) (Fig. S6 $A$ and Table S3).

Proteins Induced by Telomere Dysfunction and DNA Damage Show Increased Expression in Human Diseases Associated with Telomere Shortening. Several human diseases have been associated with accelerated telomere shortening, including cirrhosis, which represents the end stage of chronic liver diseases leading to organ failure (15). mRNA expression of the identified markers was significantly increased in liver biopsies from patients with advanced liver cirrhosis $(n=25$; mean age, $57 \pm 6.5$ years $)$ compared with biopsies from precirrhotic liver of age-matched patients with chronic liver disease $(n=20$; mean age, $54 \pm 3.2$ years) (Fig. S7A). Similarly, plasma levels of the marker proteins and chitinase enzyme activity were up-regulated in cirrhosis patients $(n=33$; mean age, $58 \pm 9.1$ years) compared with age-matched, noncirrhotic patients $(n=26$; mean age, $56 \pm 8.4$ years) (Fig. S7 $B-E$ ). Multivariate analysis revealed that EF- $1 \alpha$ and stathmin protein levels and chitinase enzyme activity had the best discriminatory power (combination score $=1.916 \times \mathrm{EF}-1 \alpha+2.607 \times$ stathmin $+0.029 \times$ chitinase $)$ (Table S4) and the combination of these three markers had the highest statistical power to discriminate the two groups (Fig. S7 F and $G)$. IHC confirmed the up-regulation of CRAMP, EF- $1 \alpha$, and stathmin protein levels in cirrhosis compared with noncirrhotic liver (Fig. S6B).

Myelodysplastic syndromes (MDS) represent another group of diseases that are associated with telomere shortening and lead to a decline in hematopoietic stem-cell function and bonemarrow failure (29). Patients with MDS ( $n=26$; mean age, $69 \pm$ 8.3 years) showed elevated plasma levels of all four marker proteins compared with protein levels in unaffected, aged human individuals living in an elderly home $(n=20$; mean age, $85 \pm 8.1$ years) (Fig. S7 $H-K$ ). Multivariate analysis revealed that stathmin-protein level had the best discriminatory power and the combination with other markers had no additional benefit (Table S5 and Fig. S7L). The analysis of a blinded, unrelated test 
set of plasma samples confirmed the discriminatory power of the optimized marker combinations (Table S6).

\section{Discussion}

This study identified secreted proteins that are associated with the aging of telomere-dysfunctional mice. The expression of these marker proteins was not associated with the aging of wild-type mice or long-lived mice that carry mutations in the growth-hormone-receptor signaling axis. These data indicate that the identified markers were most specific for the aging of mice with dysfunctional telomeres. The results suggest that telomere dysfunction may not play a major role in the aging of mTerc $^{+/+}$laboratory mouse strains at the age of 12-24 months. This interpretation stands in agreement with the observation that laboratory mice have long telomere reserves and express readily detectable levels of telomerase activity in various tissues throughout life (30).

This study shows that the proteins that are overexpressed in aging telomere dysfunctional mice are also up-regulated in presenescent human cells in culture. In addition, the expression of these proteins increases in early passage human cells in response to $\gamma$-irradiationinduced DNA damage. These data are in line with the observations that $(i)$ human cells accumulate low levels of telomere dysfunction at the presenescent stage (18) and (ii) dysfunctional telomeres induce DNA-damage responses similar to the responses induced by $\gamma$-irradiation (31-34). The current study suggests that telomere dysfunction and $\gamma$-irradiation induces secretion of an overlapping set of proteins.

The study shows that the expression of proteins induced by telomere dysfunction or DNA damage increases during human aging. The expression of marker proteins was higher in geriatric patients compared with unaffected older individuals, suggesting that the load of DNA damage and telomere dysfunction correlates with aging-associated disease. In line with this interpretation, the biomarkers also increased in patients with cirrhosis and myelodysplatic syndromes-diseases that have been associated with telomere shortening $(15,29)$.

It is possible that secreted proteins induced by telomere dysfunction impact the function of cells and organs during aging and chronic disease. Along these lines, it has been shown that telomere dysfunction of stromal cells can influence the differentiation of neighboring cancer cells (35) and hematopoietic stem-cell function (36). Two of the secreted factors identified in this study (CRAMP and chitinase) are activated during innate immune responses and inflammatory diseases (19-21). The activation of innate immunity is one of the hallmarks of human aging linked to cardiovascular disease, neurodegenerative disease, and decreased longevity (37, 38). The current study suggests that telomere dysfunction and DNA damage may contribute to the activation of innate immunity during human aging.

In summary, the current study shows that human aging is characterized by an up-regulation of secreted proteins induced by telomere dysfunction and DNA damage. These findings are in agreement with previous studies showing that telomere dysfunction humanizes aging phenotypes in mice $(39,40)$. The newly identified biomarkers represent powerful biomarkers of human aging and diseases associated with telomere shortening. Such biomarkers could be of clinical use to improve personalized therapies in the elderly, especially in clinical settings in which invasive therapies are planned that require the regenerative capacity of older individuals (e.g., surgery, chemotherapy, or irradiation).

\section{Materials and Methods}

Mice. Mice were maintained in the pathogen-free animal facility of Hannover Medical School and fed standard rodent chow. All mice were of a C57BL/6J background.
Medium. Culture medium for peptidomic analysis was a modification of Iscove's Modified Dulbecco's Medium containing sodium bicarbonate instead of Hepes buffer.

Bone Marrow Preparation. Total bone marrow was flushed from femurs and tibias. After three washes in PBS, the bone marrow cells were plated at a density of $8 \times 10^{6} / \mathrm{ml}$ of medium in a 24-well plate. Serum-free culture medium was supplemented with $10 \mu \mathrm{g} / \mathrm{ml}$ Z-VAD (OMe)-FMK, a Caspase family inhibitor (Alexis Biochemicals). Cells were cultured at $37^{\circ} \mathrm{C}$ and $5 \% \mathrm{CO}_{2}$ for $4 \mathrm{~h}$.

Supernatant Collection and Preparation. Cells were removed by spinning twice at $2,000 \times g$ for $2 \mathrm{~min}$. The supernatants were stored at $-80^{\circ} \mathrm{C}$ before proteomics analysis. Immediately before preparation, sample aliquots were thawed and 0.5 $\mathrm{ml}$ sample was desalted by using a NAP-10 gel filtration column (GE Healthcare Bio Sciences) to remove electrolytes and thereby decrease matrix effects. The sample was lyophilized in a Christ Speed-Vac RVC 2-18/Alpha1-2 (Christ) and stored at $4^{\circ} \mathrm{C}$ until use. Shortly before CE-MS analysis, the samples were resuspended in HPLC-grade $\mathrm{H}_{2} \mathrm{O}$ and the protein concentration was adjusted to 10 $\mu \mathrm{g} / \mu \mathrm{L}$ by using bicinchiconic acid assays (Interchim). Samples were injected hydrodynamically with 2 psi for $99 \mathrm{sec}$, resulting in injection volumes of $\approx 280 \mathrm{~nL}$ per sample and a total protein load of $2.8 \mu \mathrm{g}$.

CE-TOF-MS. CE-TOF-MS analysis was performed as described in ref. 41. The performance of the sample preparation procedure and the analytical performance of the instrumental setup was evaluated. The average recovery of the sample preparation procedure is $>85 \%$ with a detection limit of $\approx 1 \mathrm{fmol}$. The monoisotopic mass signals could be resolved for $z \leq 6$. The mass accuracy of the CE-TOF-MS method was determined to be $<25$ ppm for monoisotopic resolution and $<100$ ppm for unresolved peaks $(z>6)$.

Data Processing and Cluster Analysis. Data processing and cluster analysis were performed as previously described for human urine samples (41). Briefly, signals observed in a minimum of three consecutive spectra with a minimum signal-to-noise ratio of 4 were considered. After charge-state deconvolution by using MosaiquesVisu software (Biomosaiques Software) (41), the CE migration times of detected polypeptides were calibrated referring to an array of approximately 100 polypeptides that were present in all samples with high signal intensity. Total signal intensity was normalized according to total protein content. All detected polypeptides were deposited, matched, and annotated in a Microsoft SQL database (41).

Biomarker Definition and Machine Learning. As-training-set supernatants of cultured bone marrow cells of 2- and 12-month-old mTerc ${ }^{+1+}$ and $\mathrm{G}_{4 \mathrm{mTerc}}{ }^{-1-}$ mice were analyzed ( $n=5$ mice per group). Supernatants of five different subjects per group were pooled, and five individual aliquots of each pool were analyzed resulting in 20 CE-MS datasets. To enable biomarker definition, balanced two-way ANOVA was applied. Only polypeptides that were present in at least four of five replicates with median signal amplitude $>200$ counts (signalto-noise ratio $>4$ ) were analyzed. Obtained discriminating polypeptides with $P<$ 0.05 as significance level were analyzed by using ROC statistics. The obtained area-under-curve values were used as the ranking parameter to define best predictors. For machine learning, support vector machine algorithms were applied by using MosaCluster software (Biomosaiques Software) (41).

Sequencing Using LC-MS/MS. Peptides were analyzed by one-dimensional capillary liquid chromatography and tandem MS using a C18 trap column (Zorbax $300 \mathrm{SB}, 0.3 \times 50 \mathrm{~mm}$, Agilent Technologies) connected to a Magic C18 separation column $(0.1 \times 100 \mathrm{~mm}$, Thermo Scientific). The peptides were injected and trapped on the Zorbax column and eluted onto the separation column with a linear 75 -min gradient from 2 to $75 \%$ B $(0.1 \%$ acetic acid in $80 \%$ acetonitrile) in solvent $\mathrm{A}(0.1 \%$ acetic acid in $2 \%$ acetonitrile). The flow was delivered with a Rheos $2200 \mathrm{HPLC}$ system (Thermo Scientific) at $50 \mu \mathrm{l} / \mathrm{min}$. A precolumn splitter reduced the flow to $\approx 500 \mathrm{nl} / \mathrm{min}$. The eluting peptides were ionized by a Finnigan nanospray ionization source (Thermo Scientific). The LTQ Orbitrap instrument was operated in the data-dependent mode. A survey scan was performed in the Orbitrap between $\mathrm{m} / \mathrm{z} 400-1600 \mathrm{Da}$ at $60,000 \mathrm{~m}: \Delta \mathrm{m}$ resolution. The three most abundant ions detected were fragmented in the LTQ mass spectrometer and mass analyzed in the Orbitrap at a resolution of 7,500 m: $\Delta \mathrm{m}$. Singly charged ions were subjected or not to fragmentation. The normalized collision energy was set to $35 \%$.

Identification of Peptide Sequences. MS/MS data were submitted to MASCOT (www.matrixscience.com) for a search against human entries in the MDSB Protein Database. Accepted parent ion-mass deviation was 50 ppm; accepted fragment ion-mass deviation was $500 \mathrm{ppm}$. All search results with a MASCOT peptide score 
better than 20, depending on the ion coverage as related to the main spectra features, were accepted. The number of basic and neutral polar amino acids of obtained peptide sequences was used to correlate peptide sequencing data to CE-MS data as described in ref. 42.

Human Samples. The studies on human samples were conducted after the declaration of Helsinki. All samples were used anonymously. All patients and normal human controls have given written consent to provide blood or organ samples.

mRNA Gene Expression. Total RNA of organ samples was isolated by using TRIzol (Invitrogen). Transcription into CDNA was performed by using SuperScript III (Invitrogen). TaqMan Gene Expression Assays were purchased from Applied Biosystems [for mouse samples: CRAMP (Mm00438285), EF-1 $\alpha$ (Mm01966109_ul), stathmin (Mm02601853_gl), Chi3L3 (00657889_mH), and GAPDH control reagents (VIC Probe, Part No. 4308313); for human samples: CRAMP (Hs00189038_m1), EF-1 $\alpha$ (Hs00265885_g1), stathmin (Hs01027516_g1), chitinase (Hs00757767_m1), and GAPDH (Hs99999905_m1)]. The reaction mix (Absolute QPCR ROX Mix) was purchased from ABgene. All PCRs were carried out in duplicate for each CDNA sample in the ABI PRISM 7300 Sequence Detection System (Applied Biosystems).

Direct Enzyme-Linked Immunosorbent Assay (ELISA). Microwell plates (NUNC) were coated with $100 \mu \mathrm{l}$ of plasma (2-times dilution) and standard proteins: CRAMP (R.L. Gallo, University of California, San Diego) $100 \mathrm{ng}, 50 \mathrm{ng}, 25 \mathrm{ng}, 12.5$ ng, $6.25 \mathrm{ng}, 3.125 \mathrm{ng}, 1.6 \mathrm{ng}, 0 \mathrm{ng}$ at $4^{\circ} \mathrm{C}$ overnight. The coated plates were washed three times with PBS-Tween wash solution and then incubated with the first antibody [CRAMP (R.L. Gallo, University of California, San Diego) 1:1000, EF-1 $\alpha$ (Upstate) 1:1000, Chi3L3 (Shioko Kimura, National Institutes of Health) 1:1000, and stathmin (Cell Signaling Technology) 1:1000] either overnight at $4^{\circ} \mathrm{C}$ or for $2 \mathrm{~h}$ at room temperature (RT). After washing the plates three times with PBS-Tween, the assay was performed by using $100 \mu$ l of HRP-hapten conjugate secondary antibody incubated at RT for $1 \mathrm{~h}$. After five washes with PBS-Tween, $100 \mu$ l of tetramethyl benzidine peroxide-based substrate solution was added to each well, and $30 \mathrm{~min}$ later, $50 \mu \mathrm{l}$ of $2 \mathrm{M} \mathrm{H}_{2} \mathrm{SO}_{4}$ was added to each well to stop the reaction. The absorbances were read in the microplate reader in dual-wavelength mode (450-540 nm). The human CRAMP ELISA kit (HK321) was purchased from Cell Sciences, and the Chitinase assay kit (CS1030) was from Sigma.

1. Wright WE, Shay JW (1992) The two-stage mechanism controlling cellular senescence and immortalization. Exp Gerontol 27:383-389.

2. Lee HW, et al. (1998) Essential role of mouse telomerase in highly proliferative organs. Nature 392:569-574.

3. Jiang H, Ju Z, Rudolph KL (2007) Telomere shortening and ageing. Z Gerontol Geriatr 40:314-324.

4. Vulliamy T, Marrone A, Dokal I, Mason PJ (2002) Association between aplastic anaemia and mutations in telomerase RNA. Lancet 359:2168-2170.

5. Mitchell JR, Wood E, Collins K (1999) A telomerase component is defective in the human disease dyskeratosis congenita. Nature 402:551-555.

6. Tsakiri KD, etal. (2007) Adult-onset pulmonary fibrosis caused by mutations in telomerase. Proc Natl Acad Sci USA 104:7552-7557.

7. Armanios MY, et al. (2007) Telomerase mutations in families with idiopathic pulmonary fibrosis. N Engl J Med 356:1317-1326.

8. Rudolph KL, et al. (1999) Longevity, stress response, and cancer in aging telomerasedeficient mice. Cell 96:701-712.

9. Herrera $\mathrm{E}$, et al. (1999) Disease states associated with telomerase deficiency appear earlier in mice with short telomeres. EMBO $\lrcorner 18: 2950-2960$

10. Hao LY, et al. (2005) Short telomeres, even in the presence of telomerase, limit tissue renewal capacity. Cell 123:1121-1131.

11. Choudhury AR, et al. (2007) Cdkn1a deletion improves stem cell function and lifespan of mice with dysfunctional telomeres without accelerating cancer formation. Nat Genet 39:99-105.

12. Dimri GP, et al. (1995) A biomarker that identifies senescent human cells in culture and in aging skin in vivo. Proc Natl Acad Sci USA 92:9363-9367.

13. Herbig U, Ferreira M, Condel L, Carey D, Sedivy JM (2006) Cellular senescence in aging primates. Science 311:1257.

14. Jeyapalan JC, Ferreira M, Sedivy JM, Herbig U (2007) Accumulation of senescent cells in mitotic tissues of aging primates. Mech Ageing Dev 128:36-44.

15. Wiemann SU, et al. (2002) Hepatocyte telomere shortening and senescence are general markers of human liver cirrhosis. FASEB J 16:935-942.

16. Xue W, et al. (2007) Senescence and tumour clearance is triggered by $\mathrm{p} 53$ restoration in murine liver carcinomas. Nature 445:656-666.

17. Satyanarayana A, et al. (2004) Mitogen stimulation cooperates with telomere shortening to activate DNA damage responses and senescence. Mol Cell Biol 24:5459-5474.

18. Zou Y, Sfeir A, Gryaznov SM, Shay JW, Wright WE (2004) Does a sentinel or a subset of short telomeres determine replicative senescence? Mol Biol Cell 15:3709-3718.

19. Nizet V, et al. (2001) Innate antimicrobial peptide protects the skin from invasive bacterial infection. Nature 414:454-457.

20. Zhu Z, et al. (2004) Acidic Mammalian Chitinase in Asthmatic Th2 Inflammation and IL-13 Pathway Activation. Science 304:1678-1682.

21. Reese TA, et al. (2007) Chitin induces accumulation in tissue of innate immune cells associated with allergy. Nature 447:92-96.

22. Dozin B, Malpeli M, Camardella L, Cancedda R, Pietrangelo A (2002) Response of young, aged and osteoarthritic human articular chondrocytes to inflammatory cytokines: Molecular and cellular aspects. Matrix Biol 21:449-459.
Chitinase Enzyme Activity. Chitinase enzyme activity was analyzed by using the chitinase assay kit from Sigma. Each reaction needed $3 \mu$ l of plasma mixed with substrate solution (4-methylumbelliferone). Enzyme activity was determined by using fluorimetry at an excitation wavelength of $360 \mathrm{~nm}$ and an emission wavelength of $450 \mathrm{~nm}$. All samples were analyzed in triplicate.

Western Blot. Protein lysates were extracted from mouse bone marrow by using RIPA lysis buffer. Protein was subjected to $10 \%$ SDS/PAGE and detected by using antibodies against CRAMP $(1: 1,000), \mathrm{EF}-1 \alpha(1: 1,000)$, Chi3L3 $(1: 1,000)$, stathmin $(1: 1,000)$, and GAPDH (1:1000). Western-blot results were quantified by using Image J software (http://rsbweb.nih.gov/ij/). Densitometric evaluation of the blots was translated to mathematic data. SPSS 14.0 was used for statistical analyses. For each experiment, three independent Western blots were quantified.

Irradiation of Human Fibroblasts. BJ fibroblasts were $\gamma$-irradiated (4 Gy) by using a Gammatron S-80 (Siemens Medical Systems). For Western blot and RNA expression analysis, cell lysates were prepared $4 \mathrm{~h}$ after irradiation by culturing the cells in DMEM (10\% FBS). For the analysis of marker expression in culture medium, the culture supernatant was collected $4 \mathrm{~h}$ after irradiation, and the cells were cultured in DMEM [0\% FBS, supplemented an apoptosis inhibitor: $10 \mu \mathrm{g} / \mathrm{ml} \mathrm{Z-VAD} \mathrm{(OMe)-}$ FMK].

Statistical Analysis. MedCalc software package (MedCalc for Windows 8.1.1.0, MedCalc Software), SPSS 14.0, and GraphPad Prism were used for statistical analyses. The one-sample Kolmogorov-Smirnov test was used to test for normal distribution of the data. Data are expressed as the mean \pm SD or median (interquartile range) as appropriate. The unpaired Student's $t$ test was used to generate $P$ values for all of the datasets. The error bars represent SD in all figures.

Information about the performance of immunofluorescence is available in SI Methods.

ACKNOWLEDGMENTS. We thank Prof. R.L. Gallo for providing the anti-CRAMP antibody and purified CRAMP protein and Prof. S Kimura for providing the anti-Chi3L3 antibody. K.L.R. was supported by funding from the Deutsche Forschungsgemeinschaft (RU745/10-1, RU745/7-1, SFB738, KFO167), the WilhelmSander Foundation, the Fritz-Thyssen Foundation, the Deutsche Krebshilfe e.V., and the European Union projects GENINCA and TELOMARKER. This work was supported by the INCA consortium (www.inca-project.org) under the Sixth Research Framework Program of the European Union (Integrated Project LSHC-CT2005-018704) (to E.S., P.Z., J.J., and H.M)

23. Wang E, Moutsatsos IK, Nakamura T (1989) Cloning and molecular characterization of a cDNA clone to statin, a protein specifically expressed in nonproliferating quiescent and senescent fibroblasts. Exp Gerontol 24:485-499.

24. Giordano T, Kleinsek D, Foster DN (1989) Increase in abundance of a transcript hybridizing to elongation factor I alpha during cellular senescence and quiescence. Exp Geronto/24:501-513.

25. Rubin Cl, Atweh GF (2004) The role of stathmin in the regulation of the cell cycle. J Cell Biochem 93:242-250.

26. Ershler WB, Eller ET (2000) Age-associated increased interleukin-6 gene expression, latelife diseases, and frailty. Annu Rev Med 51:245-270.

27. Harris TB, et al. (1999) Associations of elevated interleukin-6 and C-reactive protein levels with mortality in the elderly. Am J Med 106:506-512.

28. Fagiolo $U$, et al. (1993) Increased cytokine production in mononuclear cells of healthy elderly people. Eur J Immunol 23:2375-2378.

29. Ohyashiki JH, etal. (1994) Telomere shortening associated with disease evolution pattern in myelodysplastic syndromes. Cancer Res 54:3557-3560.

30. Prowse KR, Greider CW (1995) Developmental and tissue-specific regulation of mouse telomerase and telomere length. Proc Natl Acad Sci USA 92:4818-4822.

31. Takai H, Smogorzewska A, de Lange T (2003) DNA damage foci at dysfunctional telomeres. Curr Biol 13:1549-1556.

32. d'Adda di Fagagna F, et al. (2003) A DNA damage checkpoint response in telomereinitiated senescence. Nature 426:194-198.

33. Chin L, et al. (1999) p53 deficiency rescues the adverse effects of telomere loss and cooperates with telomere dysfunction to accelerate carcinogenesis. Cell 97:527-538.

34. Schaetzlein S, et al. (2007) Exonuclease-1 deletion impairs DNA damage signaling and prolongs lifespan of telomere-dysfunctional mice. Cell 130:863-877.

35. Parrinello S, Coppe JP, Krtolica A, Campisi J (2005) Stromal-epithelial interactions in aging and cancer: Senescent fibroblasts alter epithelial cell differentiation. J Cell Sci 118:485-496.

36. Ju Z, et al. (2007) Telomere dysfunction induces environmental alterations limiting hematopoietic stem cell function and engraftment. Nat Med 13:742-747.

37. Vasto S, et al. (2007) Inflammatory networks in ageing, age-related diseases and longevity. Mech Ageing Dev 128:83-91.

38. Licastro F, et al. (2003) Interleukin-6 gene alleles affect the risk of Alzheimer's disease and levels of the cytokine in blood and brain. Neurobiol Aging 24:921-926.

39. Artandi SE, et al. (2000) Telomere dysfunction promotes non-reciprocal translocations and epithelial cancers in mice. Nature 406:641-645.

40. Chang S, et al. (2004) Essential role of limiting telomeres in the pathogenesis of Werner syndrome. Nat Genet 36:877-882.

41. Theodorescu D, et al. (2005) Pilot study of capillary electrophoresis coupled to mass spectrometry as a tool to define potential prostate cancer biomarkers in urine. Electrophoresis 26:2797-2808.

42. Zurbig $\mathrm{P}$, et al. (2006) Biomarker discovery by CE-MS enables sequence analysis via MS/MS with platform-independent separation. Electrophoresis 27:2111-2125. 